Article

\title{
Effect of Various Compounds Blocking the Colony Pigmentation on the Aflatoxin B1 Production by Aspergillus flavus
}

\author{
Vitaly G. Dzhavakhiya, Tatiana M. Voinova, Sofya B. Popletaeva, Natalia V. Statsyuk *, \\ Lyudmila A. Limantseva and Larisa A. Shcherbakova
}

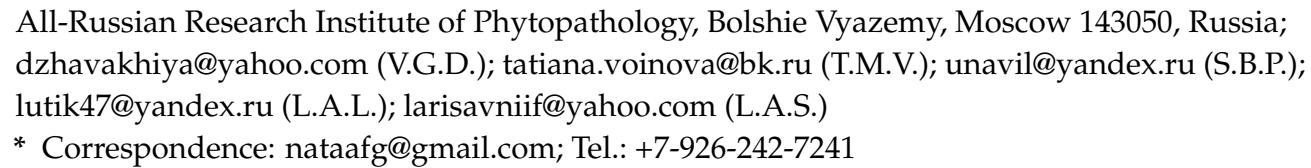

Academic Editors: Kris Audenaert, Sarah De Saeger and Siska Croubels

Received: 15 September 2016; Accepted: 24 October 2016; Published: 28 October 2016

\begin{abstract}
Aflatoxins and melanins are the products of a polyketide biosynthesis. In this study, the search of potential inhibitors of the aflatoxin B1 (AFB1) biosynthesis was performed among compounds blocking the pigmentation in fungi. Four compounds-three natural (thymol, 3-hydroxybenzaldehyde, compactin) and one synthetic (fluconazole) - were examined for their ability to block the pigmentation and AFB1 production in Aspergillus flavus. All compounds inhibited the mycelium pigmentation of a fungus growing on solid medium. At the same time, thymol, fluconazole, and 3-hydroxybenzaldehyde stimulated AFB1 accumulation in culture broth of A. flavus under submerged fermentation, whereas the addition of $2.5 \mu \mathrm{g} / \mathrm{mL}$ of compactin resulted in a $50 \times$ reduction in AFB1 production. Moreover, compactin also suppressed the sporulation of $A$. flavus on solid medium. In vivo treatment of corn and wheat grain with compactin $(50 \mu \mathrm{g} / \mathrm{g}$ of grain) reduced the level of AFB1 accumulation 14 and 15 times, respectively. Further prospects of the compactin study as potential AFB1 inhibitor are discussed.
\end{abstract}

Keywords: aflatoxin B1; fungal melanins; polyketide biosynthesis; compactin; thymol; fluconazole; 3-hydroxybenzaldehyde; Aspergillus flavus

\section{Introduction}

Fungal polyketides represent a large group of biologically active compounds synthesized by enzymes from the polyketide synthase (PKS) family [1]. Aflatoxins are secondary metabolites produced by Aspergillus flavus via the polyketide pathway. The ability to produce aflatoxins is probably not related to the essential features of A. flavus, but may give some competitive advantages to this fungus compared with other toxin-sensitive microorganisms [2,3]. A highly hepatotoxic and carcinogenic aflatoxin B1 (AFB1) is of greatest concern because it can contaminate a wide range of agricultural products that results in great losses in agricultural income and has a significant negative economic impact. A number of methods are developed for pre- and post-harvest aflatoxin management [4]. Traditional approaches, such as fungicides, have shown very limited success in the AFB1 control [5]. Therefore, the search and development of efficient preparations able to inhibit AFB1 production still remains of great theoretical and practical importance.

Fungal melanins, representing high-molecular hydrophobic pigments, are also synthesized via the PKS-depending pathway [6]. The blocking of the melanin biosynthesis in pathogenic fungi can result in the loss of their pathogenicity and an increased susceptibility of fungi to biotic and abiotic stresses [7-9]. For example, a defect in one of the PKS-encoding genes of $A$. flavus resulting in a formation of non-pigmented sclerotia significantly increased the susceptibility of the fungus to the 
deleterious effect of UV light and heat; in addition, the melanin-deficient mutant was less resistant to insect predation as compared with the wild-type strain generating melanized sclerotia [10].

Based on the data of experiments with radiolabeled polyketide intermediates, the following simplified scheme of AFB1 biosynthesis was proposed: acetate $\rightarrow$ hypothetic polyketide intermediates $\rightarrow$ norsolorinic acid $\rightarrow$ averantin $\rightarrow$ averufin $\rightarrow$ versiconal hemiacetal acetate $\rightarrow$ versicolorin A $\rightarrow$ sterigmatocystin $\rightarrow$ AFB1 [11]. Later, this scheme was significantly enlarged. To date, AFB1 biosynthesis is considered to include 23 enzymatic reactions, and 15 intermediates of this pathway have been identified [12].

Norsolorinic acid is the first stable intermediate in the aflatoxin biosynthesis. A structural similarity between the pigments of $A$. flavus spores and norsolorinic acid made it possible to suppose that these pigments and aflatoxins have common precursors; therefore, aflatoxin and melanin biosynthetic pathways have common initial stages [13]. Due to this fact, the search for compounds able to block the early stages of the polyketide biosynthetic pathway before its branching to the aflatoxin and melanin biosyntheses represented a promising task, since such inhibitors would prevent AFB1 accumulation in treated food and feed products and simultaneously decrease the contamination of these products with $A$. flavus due to the decreased viability of melanin-deficient fungus.

In our earlier studies, we tested various phosphoanalogues of amino acids and their derivatives for their ability to block different stages of the polyketide biosynthetic pathway. As a result, some compounds, which blocked either AFB1 or melanin biosynthesis, have been revealed; in the last case, a simultaneous stimulation of the toxinogenesis was observed [14,15]. However, none of these compounds was able to block early stages of polyketide biosynthesis, i.e., simultaneously inhibit the production of AFB1 and melanin.

This study continued the search for inhibitors of the early stages of toxinogenesis among natural and synthetic compounds, which, as we found earlier, are able to block the pigmentation of some plant pathogenic fungi. Our earlier study showed that compactin, a natural inhibitor of the sterol biosynthesis, which inhibits HMG-CoA reductase catalyzing the conversion of HMG-CoA into mevalonic acid [16], causes the depigmentation of colonies of several plant pathogenic fungi [17]. Another possible inhibitor of AFB1 biosynthesis could be fluconazole, a synthetic triazole-based fungicide. It is known that the fungicide action of triazoles is determined by their ability to inhibit the biosynthesis of sterols [18]. In the preliminarily study, we showed that this fungicide was able to depigment the colonies of some fungi including $A$. flavus, so we supposed it could also influence on toxinogenesis.

According to one of the existing hypotheses, the evolution of plants, which are infected with toxigenic fungi, could result in their ability to produce specific compounds inhibiting the biosynthesis of toxins [19]. In fact, some plant terpenoids are really able to suppress AFB1 production by A. flavus [20]. One of such compounds is thymol, widely used in medicine, veterinary, and plant protection, so we included this compound into this study.

It is known that 2-chloroethyl phosphoric acid inhibits AFB1 biosynthesis due to oxidative stress alleviation [21]. Some other studies showed an important role of oxidative stress at the initial stages of AFB1 biosynthesis, though the mechanisms of this effect and the specific forms of reactive oxygen influencing on the toxinogenesis still remain unclear [22]. The earlier study devoted to the chemosensitization of Aspergillus spp. and some other fungi to the action of various antifungal agents showed that sensitizers, which can act as antioxidants preventing the oxidative stress, increased the sensitivity of fungi to industrial fungicides [23]. Among the tested compounds, the most active chemosensitizer was 3-hydroxybenzaldehyde (3-HBA), a compound of a plant origin. Since the antioxidant properties of this compound could probably inhibit the toxigenesis in $A$. flavus, it was also included into the current study.

Thus, based on the above-described considerations, the purpose of this study was the examination of four compounds of different nature (Figure 1) for their effect on both AFB1 and melanin production to find compound(s) able to block the early stages of polyketide biosynthesis, as well as the in vivo 
evaluation of the AFB1 accumulation in wheat and corn grain infected with toxigenic $A$. flavus and treated with the most efficient compound.

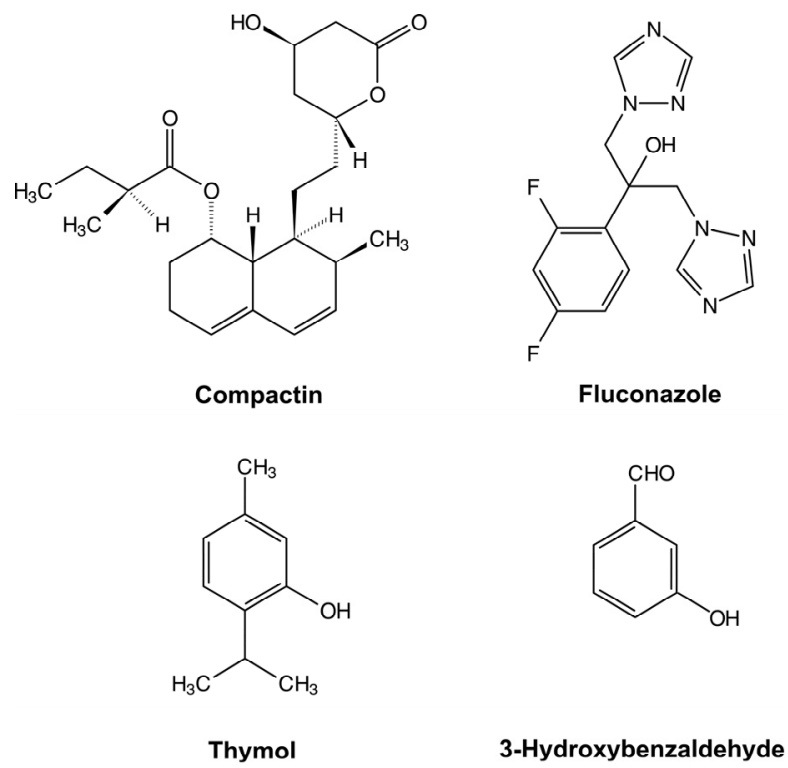

Figure 1. Compounds used in the study.

\section{Results}

\subsection{Effect of Tested Compounds on the Pigment Production in Aspergillus flavus}

According to the obtained results, all tested compounds were able to suppress the pigment production in A. flavus (Figure 2).

The maximum effect was observed for compactin; the mycelium changed its color from green to yellow starting from the compactin concentration equal to $2.5 \mu \mathrm{g} / \mathrm{mL}$, while no significant growth suppression was revealed (Figure 2, Table 1). Such a picture was observed for either stab-inoculation into the center of a Petri dish or the spreading of spore suspension on the whole agar surface. Taking into account changes in the colony diameter in the case of the stab-inoculation, the compactin concentration providing the fungal growth suppression $(25 \mu \mathrm{g} / \mathrm{mL})$ is ten times greater than the concentration, at which mycelium begins to change its color $(2.5 \mu \mathrm{g} / \mathrm{mL})$.

In the case of a stub-inoculation of control (inhibitor-free) Petri dishes, $A$. flavus grows as coalesced small pigmented colonies, which is caused by abundant sporulation accompanied by a discharge of spores onto nutrient medium with their further germination. In the case of compactin-containing medium, the situation is quite different starting from the compactin concentration equal to $25 \mu \mathrm{g} / \mathrm{mL}$. After a stub-inoculation, only one discolored colony grows in the center of a Petri dish (Figure 2). This phenomenon was observed for all compactin concentrations causing a full discoloration of mycelium $(>25 \mu \mathrm{g} / \mathrm{mL}$ ) and is explained by the inhibition of the spore generation on aerial mycelium, since the morphological examination of the mycelium under a light microscope did not reveal any spores in colonies grown on compactin-containing medium. In the case of the compactin concentration range 1-10 $\mathrm{gg} / \mathrm{mL}$, characterized by incomplete mycelium discoloration, the microscoping of colonies showed the presence of conidia and spores, but their color was clarified as compared with the control.

In the case of the $A$. flavus cultivation on the liquid compactin-containing medium, it was difficult to evaluate the effect of compactin on the mycelium pigmentation, since the fungal growth was significantly suppressed, when the compactin concentration exceeded $1 \mu \mathrm{g} / \mathrm{mL}$. The dry weight of mycelium in the control variant and at the compactin concentrations of $1,2.5$, and $5 \mu \mathrm{g} / \mathrm{mL}$ was $1.53 \pm 0.04,1.33 \pm 0.34,0.10 \pm 0.04$, and $0.12 \pm 0.03 \mathrm{~g}$, respectively; a weak discoloration was observed at the compactin concentration of $1 \mu \mathrm{g} / \mathrm{mL}$ (Figure 3 ). 
A

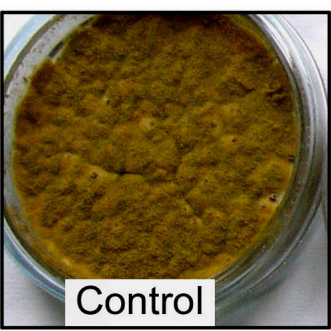

B

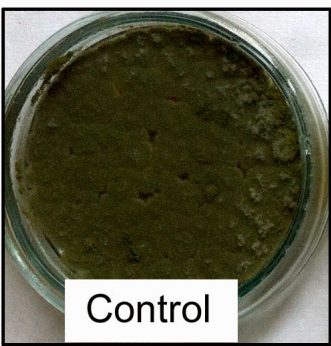

C
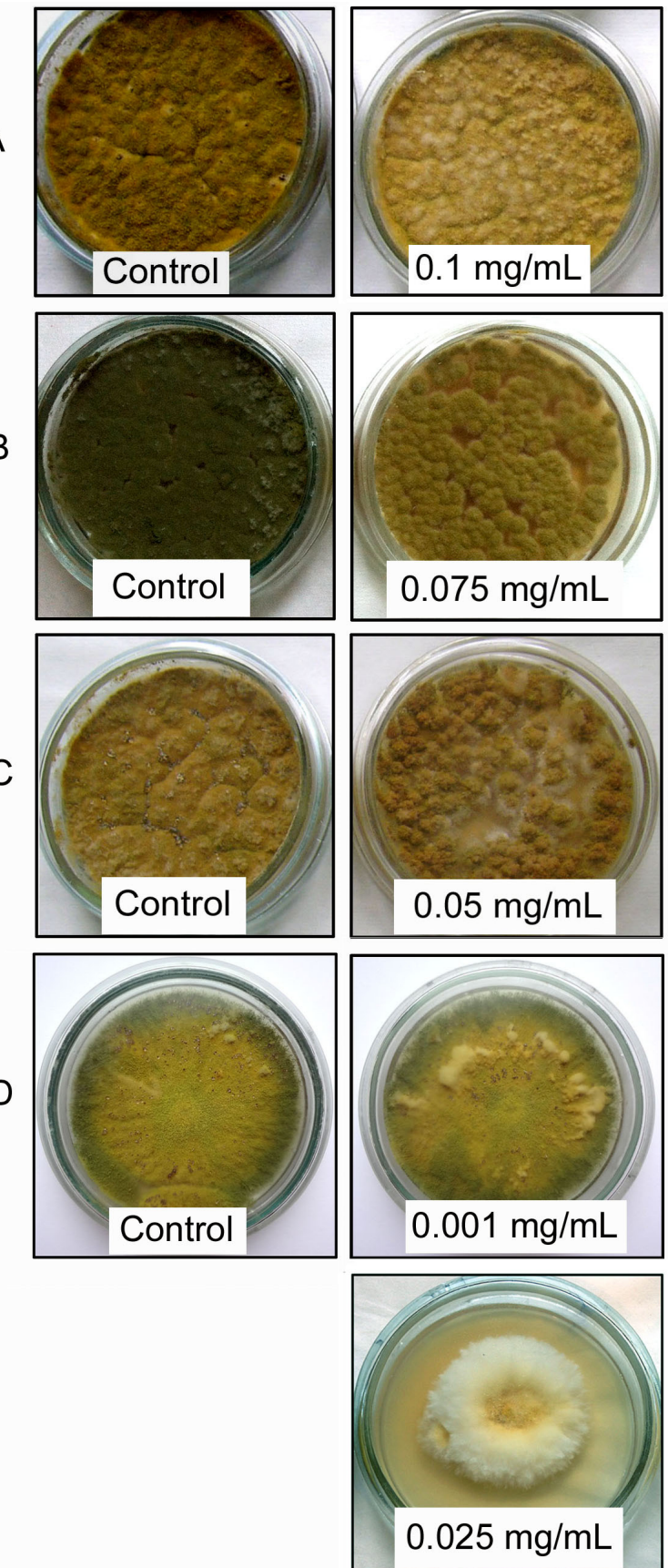
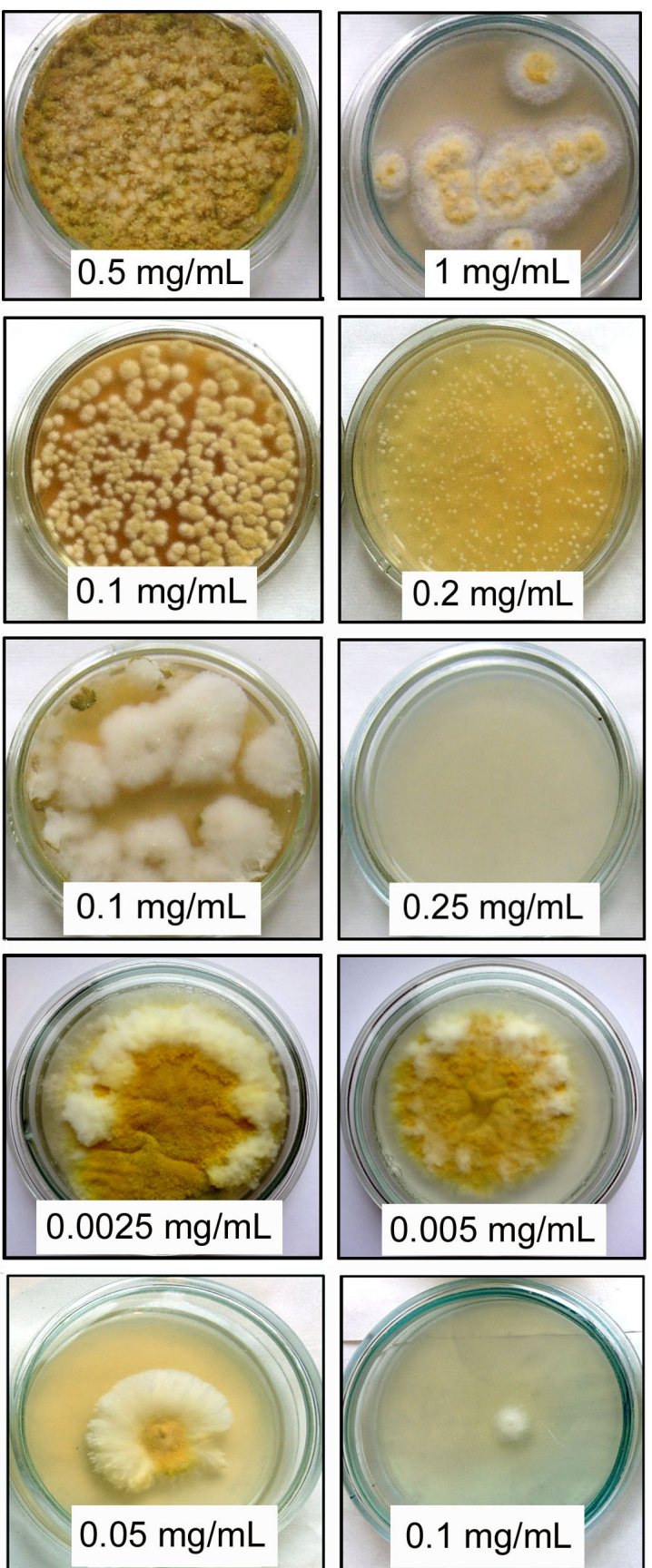

Figure 2. Effect of various compounds on the colony growth and pigmentation of Aspergillus flavus. (A) 3-hydroxybenzaldehyde; (B) thymol; (C) fluconazole; (D) compactin.

Table 1. Effect of various compactin content in agar medium on the growth of Aspergillus flavus colonies and the color of aerial mycelium.

\begin{tabular}{ccc}
\hline Compactin Concentration, $\boldsymbol{\mu g} / \mathbf{m L}$ & Colony Diameter, $\mathbf{~ m m}$ & Aerial Mycelium Color \\
\hline 0 (control) & 90 & Green \\
1 & 90 & Green \\
2.5 & 90 & Bright yellow/white \\
5 & 89 & Bright yellow/white \\
10 & 88 & Bright yellow/white \\
25 & 56 & White with yellow center \\
50 & 45 & White with yellow center \\
\hline
\end{tabular}




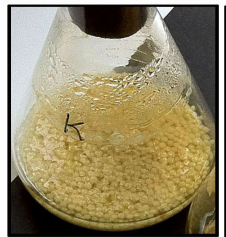

Control

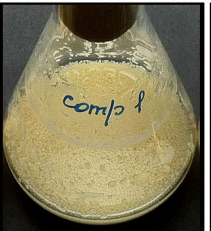

$1 \mu \mathrm{g} / \mathrm{mL}$

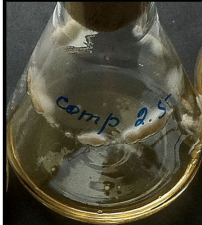

$2.5 \mu \mathrm{g} / \mathrm{mL}$

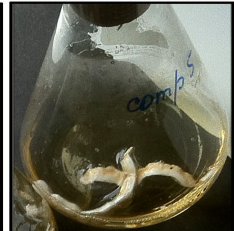

$5 \mu \mathrm{g} / \mathrm{mL}$

Figure 3. Effect of various compactin concentrations on the mycelium growth of Aspergillus flavus on liquid medium.

\subsection{Effect of Tested Compounds on the Aflatoxin Production by Aspergillus flavus}

The effect of the tested compounds on AFB1 production by A. flavus grown in liquid medium is shown in Figure 4. According to the obtained data, fluconazole fungicide added to the liquid medium at the concentrations of $50-100 \mu \mathrm{g} / \mathrm{mL}$ several times increased AFB1 production by A. flavus. An increased AFB1 production was also observed in the case of thymol and 3-HBA. Thus, the effect of these three compounds was similar to that observed earlier for $N$-hydroxyputrescine and (1-aminoethyl)phosphonic acid [24].

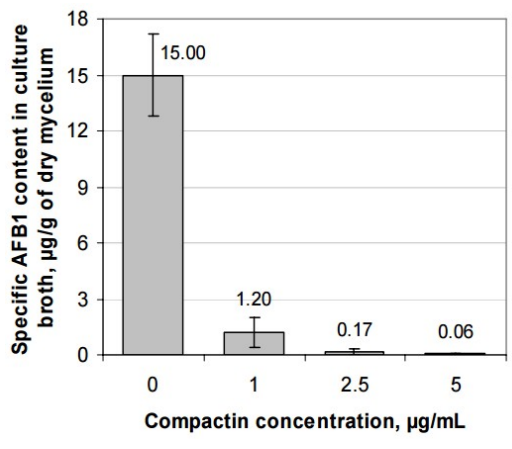

(a)

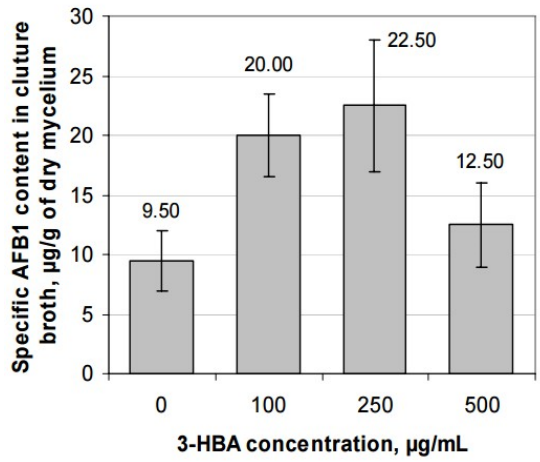

(c)

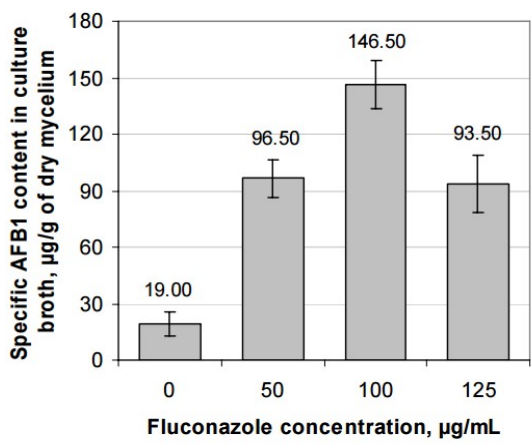

(b)

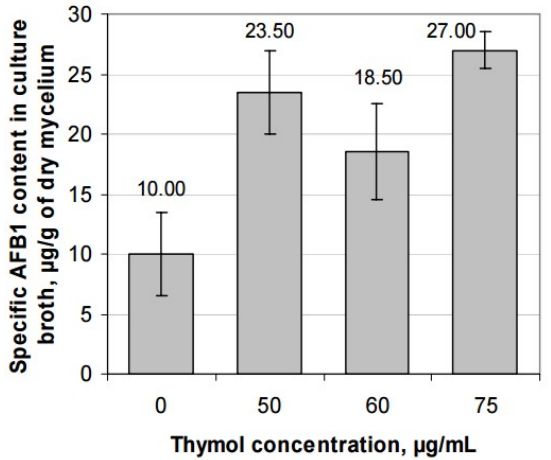

(d)

Figure 4. Effect of the tested compounds on the aflatoxin B1 production by Aspergillus flavus: (a) compactin; (b) fluconazole; (c) 3-hydroxybenzaldehyde; (d) thymol.

Unlike other compounds, compactin significantly reduced the AFB1 accumulation in culture broth The calculated coefficient of the correlation between the compactin concentration and specific AFB1 content in culture broth was -0.646 , which, according to the Chaddock scale, indicates a significant negative correlation between the mentioned parameters. Therefore, a conclusion can be made that compactin inhibits AFB1 production in A. flavus more actively than it suppresses the growth of mycelium. The experimental data confirm this conclusion: for example, the presence of $1 \mu \mathrm{g} / \mathrm{mL}$ of compactin almost did not suppress the fungal growth, while significantly reducing the toxin production (Figures 3 and 4). 


\subsection{In Vivo Evaluation of the Aflatoxin B1 Content in Treated Grain Samples}

The data on the effect of the preliminary treatment of corn and wheat grain with compactin on the AFB1 accumulation in grain infected with A. flavus are shown in Figure 5.

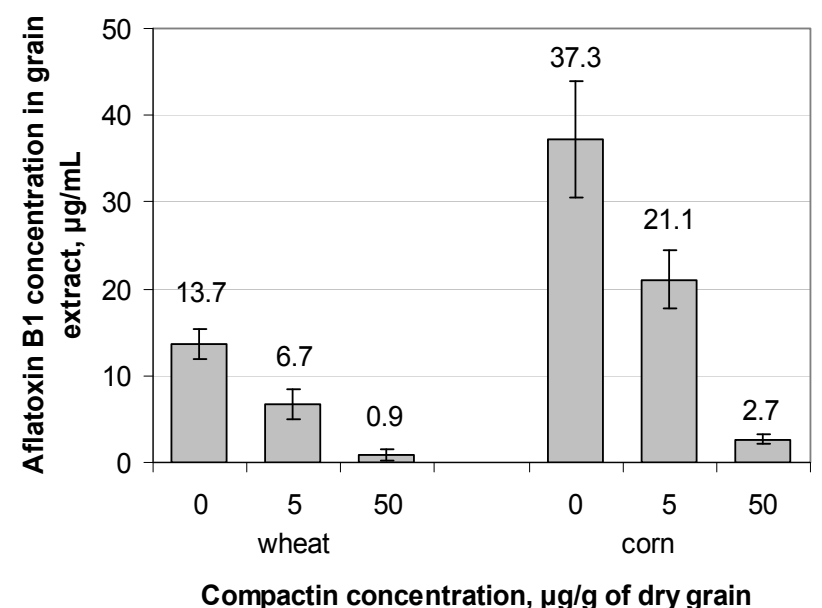

Figure 5. Aflatoxin B1 content in extracts from wheat and corn grain treated with compactin and artificially infected with toxigenic Aspergillus flavus.

In both cases, AFB1 concentration in extracts obtained from compactin-treated grain was significantly lower than that in the control reducing up to $6 \%-7 \%$ for the maximum applied compactin concentration $(50 \mu \mathrm{g} / \mathrm{g}$ of dry grain). The calculated correlation coefficients between the applied compactin concentration and the extracted AFB1 were -0.93 and -0.90 for corn and wheat, respectively.

A difference between the growth of the fungus on treated and untreated grain was clearly visible (Figure 6), especially in the case of corn. The surface of untreated grains was covered by green colonies, whereas, in the case of the treated grain, less developed white mycelium was observed, which did not contain mature pigmented conidia.

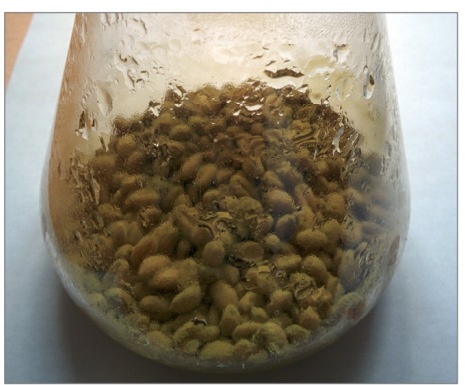

(a)

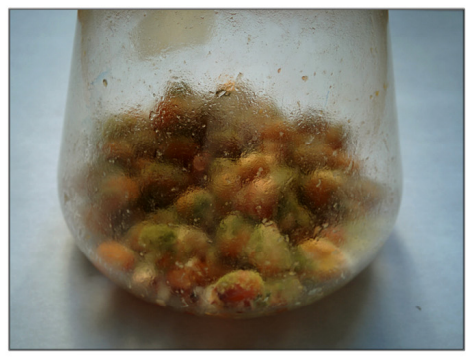

(c)

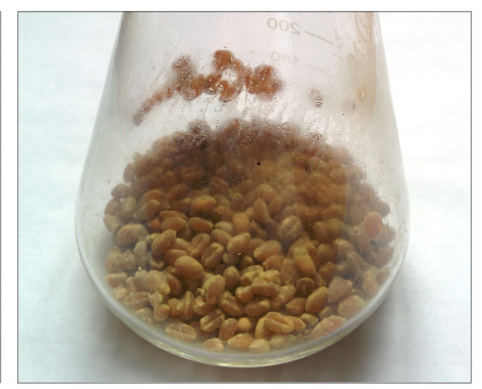

(b)

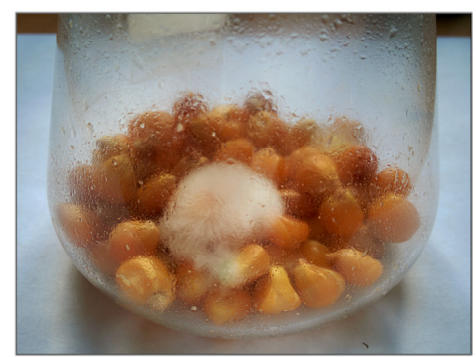

(d)

Figure 6. Development of Aspergillus flavus on grain treated with compactin ( $5 \mu \mathrm{g} / \mathrm{g}$ of dry grain): (a) control and (b) treated wheat grain; (c) control and (d) treated corn grain. 


\section{Discussion}

To date, there have been many studies describing various natural and synthetic compounds that have been shown to be specific inhibitors of AFB1 production. The majority of these compounds are of plant origin $[20,25]$. There are also some microbial metabolites, such as the melanin pigment from Streptomyces torulosis [26], some other metabolites from Streptomyces sp. [27], and respiration inhibitors of a fungal origin, characterized by the ability to significantly reduce AFB1 production by A. flavus without any significant effect on the fungal colony growth [28]. It is also known that some synthetic inhibitors of the pentaketide biosynthesis of melanin, such as the tricyclazole fungicide, are also able to inhibit AFB1 biosynthesis presumably via the inhibition of reductase involved in the versicolorin A conversion to dimethyl-sterigmatostycin at the late stages of AFB1 biosynthesis [29].

In this and previous studies, we examined a hypothesis about the possibility to reveal effective inhibitors of the early stages of AFB1 biosynthesis among compounds able to block the pigmentation of the fungus. As far as the authors know, compounds able to simultaneously inhibit both melanin and AFB1 production have only been described in a few publications [29,30], but no any special study of melanogenesis inhibitors was carried out.

Three of four compounds tested in this study suppressed the biosynthesis of the fungal pigment and, at the same time, stimulated the toxinogenesis. We also revealed several such compounds in our previous studies [14,24]. This effect can be explained by the common initial biosynthetic stages of both metabolites first hypothesized by Brown and Salvo [13]. At the later stages, the polyketide biosynthetic chain may branch in different pathways resulting in the production of melanin and AFB1. In this case, the observed stimulation of AFB1 production may be explained in the following way: these compounds block the melanin production at the stages located after the branching point that promotes the accumulation of preceding intermediates, which are also AFB1 precursors. An increased precursor accumulation provides increased AFB1 production.

The last tested compound, compactin, was able to suppress both pigment and toxin formation. One of the possible explanations for this is that it blocks the polyketide biosynthetic chain prior to the branching point; another possibility is that compactin is able to simultaneously block both melanin and AFB1 biosynthetic chains after the branching point. A more detailed study is required to determine if any of these variants is correct.

All inhibitors of the colony pigmentation tested in our current and previous studies showed either a stimulating or an inhibiting effect on AFB1 biosynthesis. Therefore, our data probably confirms the interrelation between the aflatoxinogenesis and the melanin biosynthesis in A. flavus occurring via the DOPA pathway [6] and can be used for the further elucidation of the early stages of toxinogenesis. The revealed interrelation can probably be used for practical purposes: the examination of potential AFB1 inhibitors for their ability to block melanin production can serve as a preliminary screening stage able to reveal highly effective toxin inhibitors, such as compactin. In addition, efficient AFB1 inhibitors could be probably revealed among the known inhibitors of the melanin biosynthesis.

The analysis of existing scientific and patent publications showed a lack of any information about the ability of compactin or other statins to inhibit the biosynthesis of mycotoxins. Therefore, we probably revealed a new group of AFB1 inhibitors that opens wide prospects for future studies. Among the planned theoretical tasks, we can mention the determination of the exact point of compactin action in the polyketide biosynthetic pathway using known intermediates and the study of the compactin effect on other mycotoxins of the polyketide origin, such as zearalenone. The authors also plan to continue the study of other statins for their potential as AFB1 inhibitors and have already obtained promising results for lovastatin [24]. Note that, according to the obtained data, compactin is able to inhibit the toxinogenesis at very low concentrations. Finally, an in vivo study of the compactin ability to efficiently reduce AFB1 accumulation in wheat and corn grain contaminated with toxigenic $A$. flavus demonstrated a potential for the practical application of this compound. Since the treatment of feed using such concentrations of active substances does not require high-purity compactin, the technology of its isolation and purification from culture broth can be simplified to 
reduce the cost of the final product. According to our calculations, the cost of such partially purified compactin obtained using our overproducing strain should be about $50 \mathrm{USD} / \mathrm{kg}$. Taking into account the working compactin dose of $0.1 \mathrm{mg} / \mathrm{kg}$ of grain, the final cost of the grain treatment with compactin will make about 5 USD/ton.

In our further work, we plan to study some practical aspects of the possible statin application as AFB1 inhibitors for feed treatment, such as the evaluation of the duration of their inhibiting effect and toxicity assay towards birds and mammals.

\section{Materials and Methods}

\subsection{Strains, Chemicals, and Culture Conditions}

Aspergillus flavus AF11 (VKM F-27) was used as a producer of AFB1 throughout the study. The strain was purchased in the All-Russian Collection of Microorganisms (Skryabin Institute of Biochemistry and Physiology of Microorganisms, Pushchino, Russia). AF11 was maintained on agar medium of the following composition (g/L): agar, 20; yeast extract, 5; glucose, 20. In addition, a microelement stock solution $\left(\mathrm{FeSO}_{4} \cdot 7 \mathrm{H}_{2} \mathrm{O}(24 \mathrm{mg} / \mathrm{mL}), \mathrm{MnCl}_{2} \cdot 4 \mathrm{H}_{2} \mathrm{O}(42 \mathrm{mg} / \mathrm{mL}), \mathrm{CuCl}_{2} \cdot 2 \mathrm{H}_{2} \mathrm{O}\right.$ $\left.(12 \mathrm{mg} / \mathrm{mL}), \mathrm{ZnSO}_{4} \cdot 7 \mathrm{H}_{2} \mathrm{O}(84 \mathrm{mg} / \mathrm{mL})\right)$ was added at a ratio of $100 \mu \mathrm{L}$ per $1 \mathrm{~L}$ of the agar medium. The same medium was used to observe mycelium color changes.

A spore suspension prepared from a 10-day-old culture at a concentration of $1 \times 10^{7}$ spores $/ \mathrm{mL}$ was used as the inoculum. Spore suspension $(1 \mathrm{~mL})$ was inoculated into $250 \mathrm{~mL}$ shaken flasks containing $50 \mathrm{~mL}$ of liquid Payne-Hagler medium [31]. Before the inoculation, the medium was supplemented with solutions of tested compounds sterilized by a filtration through $0.22 \mu \mathrm{m}$ Millipore membrane filters (MilliporeSigma, St. Louis, MO, USA). In the case of control flasks, the medium did not contain potential inhibitors. The culture was incubated at $27^{\circ} \mathrm{C}$ for $170 \mathrm{~h}$ under aeration conditions on a New Brunswick ${ }^{\mathrm{TM}}$ Excella E25/25R incubation shaker (New Brunswick Scientific, Edison, NJ, USA) at $200 \mathrm{rpm}$ and a $5 \mathrm{~cm}$ orbit.

Thymol, fluconazole, and 3-HBA were purchased from Medsi LLC (Moscow, Russia), Makiz-Pharma LLC (Moscow, Russia), and Sigma-Aldrich (St. Louis, MO, USA), respectively.

Compactin was obtained using an overproducing Penicillum citrinum 18-12 strain developed by a multi-step induced mutagenesis. The cultivation of this strain and the compactin isolation and purification were carried out as described earlier [32]. Since compactin has poor water solubility, for our purposes, it was converted from the lactone form to the more soluble salt form. To do this, the known amount of compactin was dissolved in ethanol (the maximum concentration did not exceed $100 \mathrm{mg} / \mathrm{mL}$ ), heated to a boil, then mixed with the equal volume of the equimolar $\mathrm{NaOH}$ solution and cooled to the room temperature. Then, the solution was diluted to the required concentration; $\mathrm{pH}$ of the final solution should be about 7.0.

The concentration range of the tested substances included minimal working concentrations providing a significant effect on the melanin production.

\subsection{Analysis of Aflatoxin B1}

After the completion of cultivation, culture broth of each experimental or control flask was centrifuged at $8000 \mathrm{~g}$ to separate the mycelia. The mycelia were washed with $3 \mathrm{~mL}$ of distilled water twice and dried at room temperature up to a constant weight.

The AFB1 content in the supernatant of culture broth was determined by HPLC as described earlier [33]. Aflatoxin B1 purchased from Sigma-Aldrich (St. Louis, MO, USA) was used as a reference sample.

To exclude the possible effect of fungal growth inhibition by the tested compounds, their inhibiting effect was assessed by the specific AFB1 content in culture broth (AFB1 content in culture broth normalized to the dry weight of mycelium). The effect of tested compounds on AFB1 biosynthesis was determined by a comparison between experimental and control flasks. 


\subsection{Evaluation of the Aflatoxin B1 Content in Treated Grain Samples}

To assess the ability of the most efficient tested compound to prevent in vivo AFB1 accumulation in grain, wheat (var. Zlata) and corn (var. Ross $140 \mathrm{SB}$ ) grain samples were used as the model objects. Twenty grams of wheat or corn grains were placed into $250 \mathrm{~mL}$ shaken flasks containing 10 or $20 \mathrm{~mL}$ of distilled water, respectively. Flasks were autoclaved for $1 \mathrm{~h}$ under a pressure of 0.5 atmosphere. Then, $1 \mathrm{~mL}$ of a sterile compactin solution $(0.1 \mathrm{mg} / \mathrm{mL}$ or $1 \mathrm{mg} / \mathrm{mL}$ ) or sterile distilled water (control) was added into the flasks. After the mixing of the flask content, each flask was inoculated by $1 \mathrm{~mL}$ of the spore suspension of $A$. flavus containing $1 \times 10^{7}$ spores $/ \mathrm{mL}$. After the 8-day incubation at $26^{\circ} \mathrm{C}, 50 \mathrm{~mL}$ of chloroform was added to each flask. Flasks were incubated for $3 \mathrm{~h}$ at $26^{\circ} \mathrm{C}$ on a New Brunswick ${ }^{\mathrm{TM}}$ Excella E25/25R incubation shaker at $250 \mathrm{rpm}$, their content was then centrifuged for $30 \mathrm{~min}$ at $8000 \mathrm{~g}$ at room temperature to separate grain from the organic phase, and $200 \mu \mathrm{L}$ of the chloroform extract was sampled from each flask. After the chloroform evaporation, the residue was dissolved in $200 \mu \mathrm{L}$ of methanol. The AFB1 concentration was further determined by HPLC as described above.

\subsection{Data Analysis}

Measurements were analyzed using a STATISTICA v. 6.1 software (StatSoft Inc., Tulsa, OK, USA) and depicted as their mean (over at least three independent experiments) \pm standard deviation. Differences were considered significant when $p<0.05$.

Acknowledgments: This work was financially supported by the Russian Science Foundation (project No. 14-16-00150).

Author Contributions: V.G.D., T.M.V., and L.A.L. conceived and designed the experiments. T.M.V., S.B.P., and L.A.L. performed the experiments. V.G.D., T.M.V., N.V.S., and L.A.S. analyzed the data and wrote the paper.

Conflicts of Interest: The authors declare no conflict of interest.

\section{References}

1. Crawford, J.M.; Townsend, C.A. New insights into the formation of fungal aromatic polyketides. Nat. Rev. Microbiol. 2010, 8, 879-889. [CrossRef] [PubMed]

2. Cotty, P.J. Virulence and cultural characteristics of two Aspergillus flavus strains pathogenic on cotton. Phytopathology 1989, 79, 808-814. [CrossRef]

3. Cary, J.W.; Ehrlich, K.C. Aflatoxigenicity in Aspergillus: Molecular genetics, phylogenetic relationships and evolutionary implications. Mycopathologia 2006, 162, 167-177. [CrossRef] [PubMed]

4. Miller, J.D.; Schaafsma, A.W.; Bhatnagar, D.; Bondy, G.; Carbone, I.; Harris, L.J.; Harrison, G.; Munkvold, G.P.; Oswald, I.P.; Pestka, J.J.; et al. Mycotoxins that affect the North American agri-food sector: State of the art and directions for the future. World Mycotoxin J. 2014, 7, 63-82. [CrossRef]

5. Abbas, H.K.; Wilkinson, J.R.; Zablotowicz, R.M.; Accinelli, C.; Abel, C.A.; Bruns, H.A.; Weaver, M.A. Ecology of Aspergillus flavus, regulation of aflatoxin production, and management strategies to reduce aflatoxin contamination of corn. Toxin Rev. 2009, 28, 142-153. [CrossRef]

6. Pal, A.K.; Gajjar, D.U.; Vasavada, A.R. DOPA and DHN pathway orchestrate melanin synthesis in Aspergillus species. Med. Mycol. 2014, 52, 10-18. [PubMed]

7. Dzhavakhiya, V.G.; Aver'yanov, A.A.; Minaev, V.I.; Ermolinskiy, B.S.; Voinova, T.M.; Lapikova, V.P.; Petelina, G.G.; Vavilova, N.A. Structure and function of cell wall melanin of the micromycete Pyricularia oryzae Cav., the rice blast pathogen. Zh. Obshch. Biol. 1990, 51, 528-535.

8. Hamada, T.; Asanagi, M.; Satozawa, T.; Araki, N.; Banba, S.; Higashimura, N.; Akase, T.; Hirase, K. Action mechanism of the novel rice blast fungicide tolprocarb distinct from that of conventional melanin biosynthesis inhibitors. J. Pestic. Sci. 2014, 39, 152-158. [CrossRef]

9. Takagaki, M. Melanin biosynthesis inhibitors. In Fungicide Resistance in Plant Pathogens; Takagaki, M., Ishii, H., Hollomon, D.W., Eds.; Springer: Tokyo, Japan, 2015; pp. 175-180. 
10. Cary, J.W.; Harris-Coward, P.Y.; Ehrlich, K.C.; Di Mavungu, J.D.; Malysheva, S.V.; De Saeger, S.; Dowd, P.F.; Shantappa, S.; Martens, S.L.; Calvo, A.M. Functional characterization of a veA-dependent polyketide synthase gene in Aspergillus flavus necessary for the synthesis of asparasone, a sclerotium-specific pigment. Fungal Genet. Biol. 2014, 64, 25-35. [CrossRef] [PubMed]

11. Bennett, J.W.; Christensen, S.B. New perspectives on aflatoxin biosynthesis. Adv. Appl. Microbiol. 1983, 29, 53-92. [PubMed]

12. Yu, J.; Chang, P.-K.; Ehrlich, K.C.; Cary, J.W.; Bhatnagar, D.; Cleveland, T.E.; Payne, G.A.; Linz, J.E.; Woloshuk, C.P.; Bennet, J.W. Clustered pathway genes in aflatoxin biosynthesis. Appl. Environ. Microbiol. 2004, 70, 1253-1262. [CrossRef] [PubMed]

13. Brown, D.W.; Salvo, J.J. Isolation and characterization of sexual spore pigments from Aspergillus nidulans. Appl. Environ. Microbiol. 1994, 60, 979-983. [PubMed]

14. Dzhavakhiya, V.; Statsyuk, N.; Shcherbakova, L. Blocking of some stages of melanogenesis can enhance aflatoxin B1 production in Aspergillus flavus. In Proceedings of Conference abstracts of the 37th Mycotoxin Workshop, Bratislava, Slovakia, 1-3 June 2015; Slovak University of Technology: Bratislava, Slovakia, 2015 ; p. 81.

15. Khomutov, R.M.; Dzhavakhiya, V.G.; Khurs, E.N.; Osipova, T.I.; Shcherbakova, L.A.; Zhemchuzhina, N.S.; Mikituk, O.D.; Nazarova, T.A. Chemical regulation of mycotoxin biosynthesis. Dokl. Biochem. Biophys. 2011, 436, 25-28. [CrossRef] [PubMed]

16. Ukraintseva, S.N.; Pridannikov, M.V.; Dzhavakhiya, V.G. Compactin: A potential biopesticide. Zas. Karantin. Rast. 2008, 2, 63. (In Russian)

17. Endo, A. The discovery and development of HMG-CoA reductase inhibitors. Lipid Res. 1978, 33, 1569-1582. [CrossRef] [PubMed]

18. Odds, F.C.; Brown, A.J.P.; Gow, N.A.R. Antifungal agents: Mechanisms of action. Trends Microbiol. 2003, 11, 272-279. [CrossRef]

19. Gonsales, E.; Felicio, J.D.; Pinto, M.M. Biflavonoids inhibit the production of aflatoxin by Aspergillus flavus. Braz. J. Med. Biol. Res. 2001, 34, 1453-1456.

20. Holmes, R.A.; Boston, R.S.; Payne, G.A. Diverse inhibitors of aflatoxin biosynthesis. Appl. Microbiol. Biotechnol. 2008, 78, 559-572. [CrossRef] [PubMed]

21. Huang, J.Q.; Jiang, H.F.; Zhou, Y.Q.; Lei, Y.; Wang, S.-Y.; Liao, B.-S. Ethylene inhibited aflatoxin biosynthesis in due to oxidative stress alleviation and related to glutathione redox state changes in Aspergillus flavus. Int. J. Food Microbiol. 2009, 130, 17-21. [CrossRef] [PubMed]

22. Grintzalis, K.; Vernardis, S.I.; Klapa, M.I.; Georgiou, C.D. Role of oxidative stress in sclerotial differentiation and aflatoxin B1 biosynthesis in Aspergillus flavus. Appl. Environ. Microbiol. 2014, 80, 5561-5571. [CrossRef] [PubMed]

23. Campbell, B.C.; Chan, K.L.; Kim, J.H. Chemosensitization as a means to augment commercial antifungal agents. Front. Microbiol. 2012, 3. [CrossRef] [PubMed]

24. Dzhavakhiya, V.G.; Voinova, T.M.; Popletaeva, S.B.; Statsyuk, N.V.; Mikityuk, O.D.; Nazarova, T.A.; Shcherbakova, L.A. Some natural and synthetic compounds inhibiting the biosynthesis of aflatoxin B1 and melanin in Aspergillus flavus. Agric. Biol. 2016, 51, 533-542. [CrossRef]

25. Zhou, W.; Hu, L.B.; Zhao, Y.; Wang, M.Y.; Zhang, H.; Mo, H.Z. Inhibition of fungal aflatoxin B1 biosynthesis by diverse botanically-derived polyphenols. Trop. J. Pharm. Res. 2015, 14, 605-609. [CrossRef]

26. Shaaban, M.T.; El-Sabbagh, S.M.M.; Alam, A. Studies on an actinomycete producing a melanin pigment inhibiting aflatoxin B1 production by Aspergillus flavus. Life Sci. J. 2013, 10, 1437-1448.

27. Sakuda, S. Mycotoxin production inhibitors from natural products. JSM Mycotoxins 2010, 60, 79-86. [CrossRef]

28. Sakuda, S.; Prabowo, D.F.; Takagi, K.; Shiomi, K.; Mori, M.; Omura, S.; Nagasawa, H. Inhibitory effects of respiration inhibitors on aflatoxin production. Toxins 2014, 6, 1193-1200. [CrossRef] [PubMed]

29. Wheeler, M.H.; Bhatnagar, D.; Rojas, M.G. Chlobenthiazone and tricyclazole inhibition of aflatoxin biosynthesis by Aspergillus flavus. Pest. Biochem. Physiol. 1989, 35, 315-323. [CrossRef]

30. Okamoto, S.; Sakurada, M.; Kubo, Y.; Tsuji, G.; Fujii, I.; Ebizuka, Y.; Ono, M.; Nagasawa, H.; Sakuda, S. Inhibitory effect of aflastatin A on melanin biosynthesis by Colletotrichum lagenarium. Microbiology 2001, 147, 2623-2628. [CrossRef] [PubMed] 
31. Payne, G.A.; Hagler, W.M. Effect of specific amino acids on growth and aflatoxin production by Aspergillius parasiticus and Aspergillius flavus in defined media. Appl. Environ. Microbiol. 1983, 46, 805-812. [PubMed]

32. Ukraintseva, S.N.; Voinova, T.M.; Dzhavakhiya, V.G. Obtaining the highly productive mutants Penicillium citrinum producing compactin and optimization of fermentation process in shaken flasks. In Biotechnology in Biology and Medicine; Egorov, A.M., Zaikov, G., Eds.; Nova Science Publishers: New York, NY, USA, 2006; pp. 233-241.

33. Shcherbakova, L.A.; Statsyuk, N.V.; Mikityuk, O.D.; Nazarova, N.A.; Dzhavakhiya, V.G. Aflatoxin B1 degradation by metabolites of Phoma glomerata PG41 isolated from natural substrate colonized by aflatoxigenic Aspergillus flavus. Jundishapur J. Microbiol. 2015, 8. [CrossRef] [PubMed]

(C) 2016 by the authors; licensee MDPI, Basel, Switzerland. This article is an open access article distributed under the terms and conditions of the Creative Commons Attribution (CC-BY) license (http://creativecommons.org/licenses/by/4.0/). 